\title{
The respiratory molybdo-selenoprotein formate dehydrogenases of Escherichia coli have hydrogen: benzyl viologen oxidoreductase activity
}

Basem Soboh ${ }^{1 \dagger}$, Constanze Pinske $^{1 \dagger}$, Martin Kuhns ${ }^{1}$, Mandy Waclawek ${ }^{1}$, Christian Ihling ${ }^{2}$, Karen Trchounian $^{3}$, Armen $\operatorname{Trchounian}^{3}$, Andrea Sinz ${ }^{2}$ and Gary Sawers ${ }^{1 *}$

\begin{abstract}
Background: Escherichia coli synthesizes three membrane-bound molybdenum- and selenocysteine-containing formate dehydrogenases, as well as up to four membrane-bound [NiFe]-hydrogenases. Two of the formate dehydrogenases (Fdh-N and Fdh-O) and two of the hydrogenases (Hyd-1 and Hyd-2) have their respective catalytic subunits located in the periplasm and these enzymes have been shown previously to oxidize formate and hydrogen, respectively, and thus function in energy metabolism. Mutants unable to synthesize the [NiFe]hydrogenases retain a $\mathrm{H}_{2}$ : benzyl viologen oxidoreductase activity. The aim of this study was to identify the enzyme or enzymes responsible for this activity.

Results: Here we report the identification of a new $\mathrm{H}_{2}$ : benzyl viologen oxidoreductase enzyme activity in $E$. coli that is independent of the [NiFe]-hydrogenases. This enzyme activity was originally identified after non-denaturing polyacrylamide gel electrophoresis and visualization of hydrogen-oxidizing activity by specific staining. Analysis of a crude extract derived from a variety of $E$. coli mutants unable to synthesize any [NiFe]-hydrogenase-associated enzyme activity revealed that the mutants retained this specific hydrogen-oxidizing activity. Enrichment of this enzyme activity from solubilised membrane fractions of the hydrogenase-negative mutant FTD147 by ionexchange, hydrophobic interaction and size-exclusion chromatographies followed by mass spectrometric analysis identified the enzymes Fdh-N and Fdh-O. Analysis of defined mutants devoid of selenocysteine biosynthetic capacity or carrying deletions in the genes encoding the catalytic subunits of Fdh-N and Fdh-O demonstrated that both enzymes catalyze hydrogen activation. Fdh-N and Fdh-O can also transfer the electrons derived from oxidation of hydrogen to other redox dyes.

Conclusions: The related respiratory molybdo-selenoproteins Fdh-N and Fdh-O of Escherichia coli have hydrogenoxidizing activity. These findings demonstrate that the energy-conserving selenium- and molybdenum-dependent formate dehydrogenases Fdh-N and Fdh-O exhibit a degree of promiscuity with respect to the electron donor they use and identify a new class of dihydrogen-oxidizing enzyme.
\end{abstract}

\footnotetext{
* Correspondence: gary.sawers@mikrobiologie.uni-halle.de

† Contributed equally

'Institute for Microbiology, Martin-Luther University Halle-Wittenberg, Kurt-

Mothes-Str. 3, 06120 Halle (Saale), Germany

Full list of author information is available at the end of the article
} 


\section{Background}

Hydrogen and formate are electron donors frequently used by anaerobic microorganisms. Metabolism of hydrogen and formate is often highly interlinked in many bacteria that can oxidize both compounds. This is exemplified in the fermentative metabolism of the enterobacterium Escherichia coli where up to one third of the carbon from glucose is converted to formate; formate is then disproportionated to $\mathrm{H}_{2}$ and $\mathrm{CO}_{2}$ [1-3]. Formate can be metabolized by three membrane-associated, molybdo-seleno formate dehydrogenases (Fdh), termed Fdh-H (associated with hydrogen production), Fdh-N (induced in the presence nitrate) and Fdh-O (also detected during aerobic growth). Fdh-H is encoded by the $f d h F$ gene and together with one of the four [NiFe]hydrogenases (Hyd) of E. coli, Hyd-3, forms the hydrogen-evolving formate hydrogenlyase (FHL) enzyme complex.

Fdh-N (FdnGHI) and Fdh-O (FdoGHI) are highly related enzymes at both the amino acid sequence and functional levels $[1,4]$. They are multi-subunit oxidoreductases each comprising a large catalytic subunit (FdnG or FdoG), an electron-transfer subunit (FdnH or $\mathrm{FdoH}$ ) and a membrane-anchoring subunit (FdnI or FdoI); the latter has a quinone-binding site that allows transfer of electrons derived from formate oxidation into the respiratory chain [4-6]. Both enzymes have their respective active site located on the periplasmic face of the cytoplasmic membrane and they couple formate oxidation to energy conservation. A key feature of all three Fdh enzymes is the presence of selenocysteine, a bis-molybdopterin guanine dinucleotide (bis-MGD) cofactor and a [4Fe-4S] cluster in their respective catalytic subunit [4,7]. Although the synthesis of the Fdh-N enzyme is induced to maximal levels during growth in the presence of nitrate, the enzyme is also present at lower levels during fermentative growth $[1,5,8]$. Fdh-O is synthesized constitutively and is present at low levels aerobically, during fermentative growth and nitrate respiration $[6,9]$.

E. coli has also the coding capacity to synthesize four membrane-associated, multi-subunit Hyd enzymes, which are termed Hyd-1 through Hyd-4 [2,10]. Hyd-1, Hyd-2 and Hyd-3 have been characterized in detail. Like Fdh-N and Fdh-O, Hyd-1 and Hyd-2 have their active sites located facing the periplasm [11]. Both enzymes oxidize hydrogen and contribute to energy conservation. Due to the fact that hydrogenases catalyze the reversible oxidation of dihydrogen in vitro, the activities of all three characterized [NiFe]-hydrogenases of E. coli can be determined simultaneously in a single reaction using hydrogen as electron donor and the artificial electron acceptor benzyl viologen (BV) $[12,13]$. Moreover, the hydrogen-oxidizing activities of Hyd-1 and Hyd-2 can also be visualized after electrophoretic separation under non-denaturing conditions in the presence of detergent [12]. Because of its apparent labile nature the activity of Hyd-3 cannot be visualized after gel electrophoresis.

It was noted many years ago [14] that in non-denaturing polyacrylamide gels a slowly-migrating protein complex with a hydrogen: BV oxidoreductase enzyme activity, apparently unrelated to either Hyd-1 or Hyd-2, could be visualized after electrophoretic separation of membrane fractions derived from $E$. coli grown under anaerobic conditions. In this study, this hydrogenaseindependent enzyme activity could be identified as being catalyzed by the highly related Fdh-N and Fdh-O enzymes.

\section{Results}

Hydrogenase-independent hydrogen: BV oxidoreductase activity in E. coli membranes

Membrane fractions derived from anaerobically cultured wild-type E. coli K-12 strains such as $\mathrm{P} 4 \mathrm{X}[12,15]$ and MC4100 [16] exhibit a slowly migrating hydrogen: benzyl viologen (BV) oxidoreductase activity that cannot be assigned to either Hyd-1 or Hyd-2. Previous findings based on non-denaturing PAGE [16] estimated a size of approximately $500 \mathrm{kDa}$ for this complex. To demonstrate the hydrogenase-independent nature of this enzyme activity, extracts derived from a hypF mutant, which lacks the central hydrogenase maturase HypF and consequently is unable to synthesize active [NiFe]hydrogenases [17], retained this single slowly migrating species exhibiting hydrogen:BV oxidoreductase activity, while the activity bands corresponding to Hyd-1 and Hyd-2 were no longer visible (Figure 1). This result demonstrates that the activity of this slowly migrating band is completely unrelated to the [NiFe]-hydrogenases Hyd-1, Hyd-2, Hyd-3 or Hyd-4. Note that no active, stained bands were observed when this experiment was performed with a nitrogen gas atmosphere (data not shown).

\section{Formate dehydrogenases $\mathrm{N}$ and $\mathrm{O}$ catalyze hydrogen:BV oxidoreduction}

In order to identify the enzyme(s) responsible for this new hydrogen: BV oxidoreductase activity, the hypF deletion mutant was grown anaerobically and the membrane fraction was prepared (see Methods). The hydrogen: BV oxidoreductase activity could be released from the membrane in soluble form by treatment with the detergent Triton X-100. Enrichment of the activity was achieved by separation from contaminating membrane proteins using Q-sepharose anion exchange, phenyl sepharose hydrophobic interaction chromatography and 


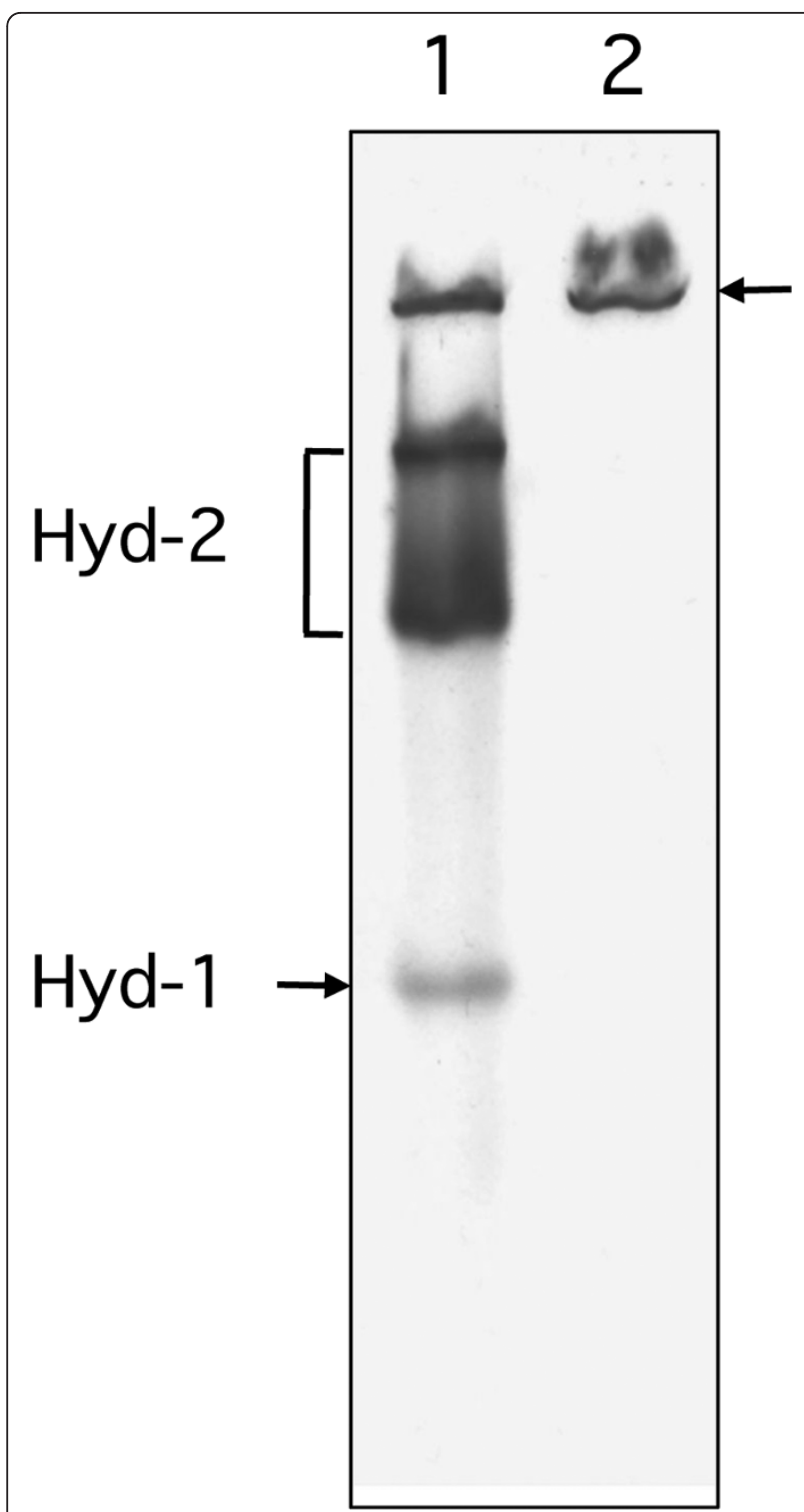

Figure 1 A hypF mutant retains hydrogenase-independent $\mathrm{H}_{2}$ : BV oxidoreductase activity. Extracts derived from MC4100 (lane 1) and the isogenic $\triangle h y p F$ mutant DHP-F2 (lane 2) were separated by non-denaturing PAGE and subsequently stained for hydrogenase enzyme activity as described in the Methods section. Strains were grown in TYEP medium with $0.8 \%(\mathrm{w} / \mathrm{v})$ glucose, $\mathrm{pH}$ 6.5. Equivalent amounts of Triton X-100-treated crude extract (50 $\mu \mathrm{g}$ of protein) were applied to each lane. The activity bands corresponding to Hyd- 1 and Hyd-2 are indicated, as is the slowly migrating activity band (designated by an arrow) that corresponds to a hydrogenaseindependent $\mathrm{H}_{2}: \mathrm{BV}$ oxidoreductase enzyme activity.

finally gel filtration on a Superdex-200 size exclusion column (see Methods for details). Fractions with enzyme activity were monitored during the enrichment procedure using activity-staining after non-denaturing PAGE. A representative elution profile from the Superdex-200 chromatography step, together with the corresponding activity gel identifying the active enzyme, are shown in Figure 2. Two distinct peaks that absorbed at $280 \mathrm{~nm}$ could be separated (Figure 2A) and the hydrogen: BV oxidoreductase activity was found to be exclusively associated with the higher molecular mass symmetric peak labelled P1 (Figure 2B). This peak eluted after 47 $\mathrm{ml}\left(\mathrm{V}_{\mathrm{o}}=45 \mathrm{ml}\right)$ and was estimated to have a mass of between 500-550 kDa (data not shown).

The band showing hydrogen: BV oxidoreductase activity in Figure 2B was carefully excised and the polypeptides within the fraction were analyzed by mass

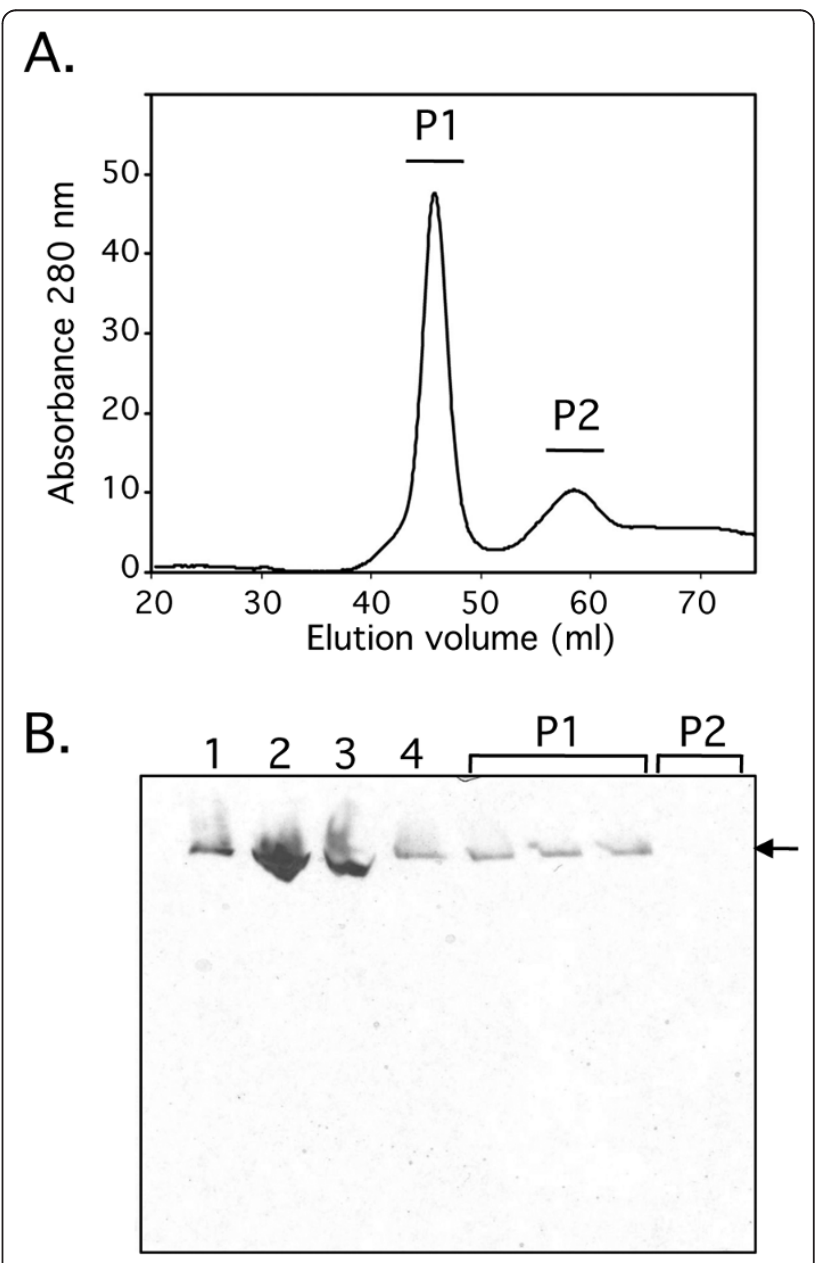

Figure 2 Chromatographic separation of the $\mathrm{H}_{2}$ : BV oxidoreductase activity on a Superdex-S200 column. A. A representative elution profile of the enriched $\mathrm{H}_{2}$ : BV oxidoreductase enzyme activity after size exclusion chromatography on SuperdexS200 is shown. The absorbance at $280 \mathrm{~nm}$ was monitored and the two main elution peaks were labelled P1 and P2. B. Samples of the fractions across the elution peaks $\mathrm{P} 1$ and $\mathrm{P} 2$ were separated by non-denaturing PAGE and subsequently stained for hydrogenase enzyme activity. Lane 1, crude cell extract (50 $\mu$ g protein); lane 2, membrane fraction ( $50 \mu \mathrm{g}$ protein); lane 3 , solubilised membrane fraction $(50 \mu \mathrm{g}$ protein); lane 4, aliquot of the $400 \mathrm{mM}$ fraction from the Q-sepharose column. The arrow identifies the $\mathrm{H}_{2}$ : BV oxidoreductase enzyme activity. 
spectrometry. Both Fdh-O and Fdh-N enzymes were unambiguously identified: the polypeptides FdoG, FdoH, FdoI, FdnG, and FdnH were identified. The catalytic subunits of Fdh-O and Fdh-N share $74 \%$ amino acid identity and both enzymes are synthesized at low levels during fermentative growth. Fdh- $\mathrm{N}$ is a trimer of trimers $(\alpha \beta \gamma)$ with molecular mass of $510 \mathrm{kDa}$ [4] and this correlates well with the estimated size of the protein complex showing $\mathrm{H}_{2}$ : BV oxidoreductase activity as revealed by non-denaturing PAGE and size exclusion chromatography.

Both Fdh-N and Fdh-O can catalyze the formatedependent reduction of either BV or DCPIP (2,6-dichlorophenolindophenol) [8,9], whereby Fdh-N transfers electrons much more readily to DCPIP than to BV [8]. Analysis of fraction P1 from the gel filtration experiment revealed a formate: $\mathrm{BV}$ oxidoreductase activity of $67 \mathrm{mU} \mathrm{mg} \mathrm{protein}{ }^{-1}$ and a formate: DCPIP oxidoreductase activity of $0.64 \mathrm{U} \mathrm{mg}$ protein $^{-1}$ (Table 1 ). In comparison, the $\mathrm{H}_{2}$ : BV oxidoreductase activity of fraction P1 was $15 \mathrm{mU}$ mg protein ${ }^{-1}$, while no enzyme activity could be detected when hydrogen gas was replaced with nitrogen gas.

All three Fdh enzymes in E. coli are selenocysteinecontaining proteins $[1,2,18]$. Therefore, a mutant unable to incorporate selenocysteine co-translationally into the polypeptides should lack this slow-migrating enzyme $\mathrm{H}_{2}$-oxidizing activity. Analysis of crude extracts derived from the selC mutant FM460, which is unable to synthesize the selenocysteine-inserting tRNA ${ }^{\text {SEC }}$ [19], lacked the hydrogenase-independent activity band observed in the wild-type (Figure 3), consistent with the activity being selenium-dependent. Notably Hyd-1 and Hyd-2 both retained activity in the selC mutant.

Fdh-N and Fdh-O can also transfer the electrons from hydrogen to other redox dyes

The catalytic subunits of Fdh-N and Fdh-O are encoded by the $f d n G$ and $f d o G$ genes, respectively $[5,6]$. To analyse the extent to which Fdh-N and Fdh-O contributed

Table 1 Activity of enriched enzyme fraction with different electron donors

\begin{tabular}{|c|c|}
\hline Electron donor and acceptor ${ }^{a}$ & $\begin{array}{l}\text { Specific Activity } \\
\left(\mathrm{mU}^{\mathrm{mg}} \text { protein }\right. \\
\end{array}$ \\
\hline $\mathrm{H}_{2}$ and benzyl viologen & $14.8 \pm 2.3$ \\
\hline Benzyl viologen without an electron donor & $<0.20$ \\
\hline Formate and benzyl viologen & $1.24 \pm 1.0$ \\
\hline Formate and PMS/DCPIP & $638.3 \pm 69$ \\
\hline
\end{tabular}

a The buffer used was $50 \mathrm{mM}$ sodium phosphate $\mathrm{pH}$ 7.2; BV was used at a final concentration of $4 \mathrm{mM}$; formate was added to a final concentration of 18 $\mathrm{mM}$; and PMS/DCPIP were added at final concentrations of $20 \mu \mathrm{M}$ and $78 \mu \mathrm{M}$, respectively.

${ }^{b}$ The mean and standard deviation $( \pm)$ of at least three independent experiments are shown.

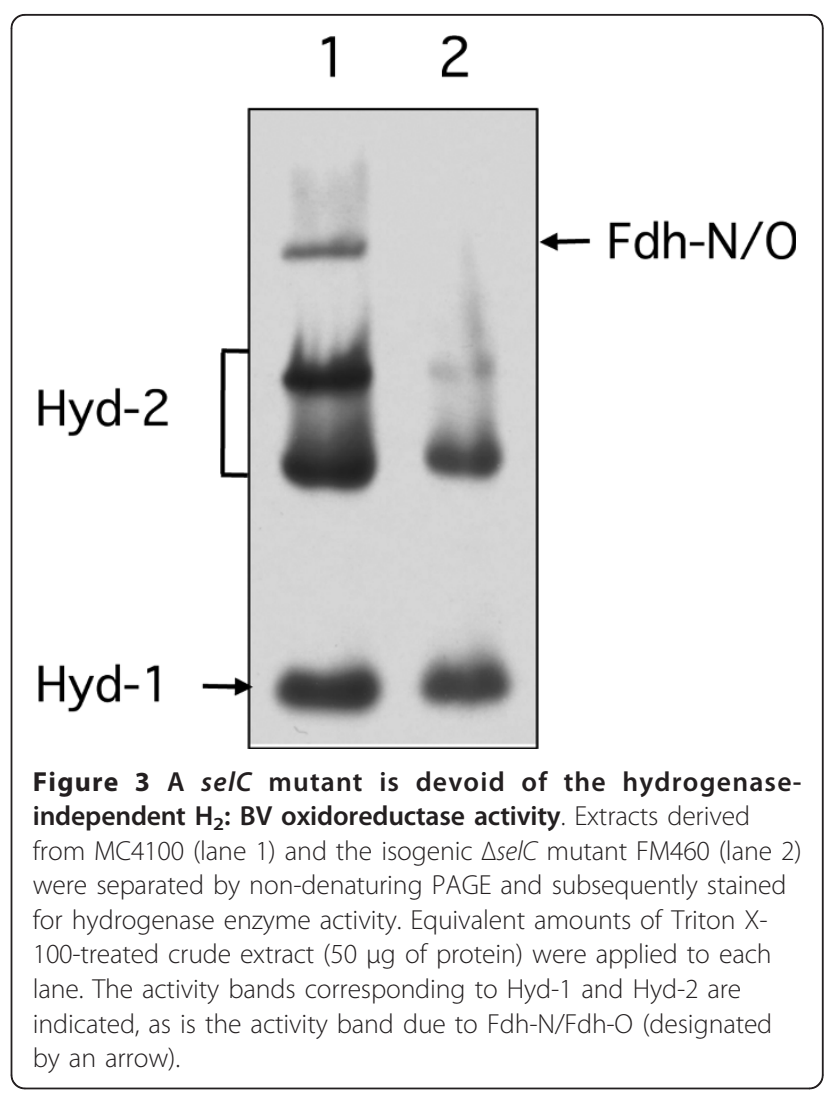

to hydrogen: BV oxidoreductase activity after fermentative growth the activity in mutants with a deletion mutation either in $f d n G$ or in $f d o G$ was analyzed. Introduction of a deletion mutation in the $f d n G$ gene resulted in a slight reduction in intensity of the $\mathrm{H}_{2}$ : BV oxidoreductase activity band (Figure $4 \mathrm{~A}$ ). The $f d o G$ mutation also resulted in a similar phenotype (Figure 4A). Introduction of the $f d n G$ or $f d o G$ genes on plasmids into the respective mutants restored full activity. An activity band associated with Hyd-2 was used as a loading control for these experiments. Strain FTD147, which has mutations in the genes encoding the catalytic subunits of Hyd-1, Hyd-2 and Hyd-3 [20], and thus cannot synthesize active [NiFe]-hydrogenases, lacked the Hyd-2 activity band but retained the Fdh-N/O hydrogen-oxidizing activity (Figure 4A top panel). Note that the isogenic wild type BW25113 of the JW series of strains had an identical phenoytype to that of MC4100 (data not shown). These experiments demonstrate that under fermentative growth conditions Fdh-N and Fdh-O both contribute to the $\mathrm{H}_{2}$ : BV oxidoreductase enzyme activity.

Fdh-N and Fdh-O catalyze the formate-dependent reduction of phenazine methosulphate/nitroblue tetrazolium (PMS/NBT), which can be used to visualize Fdh enzyme activity after non-denaturing PAGE [8]. Staining 


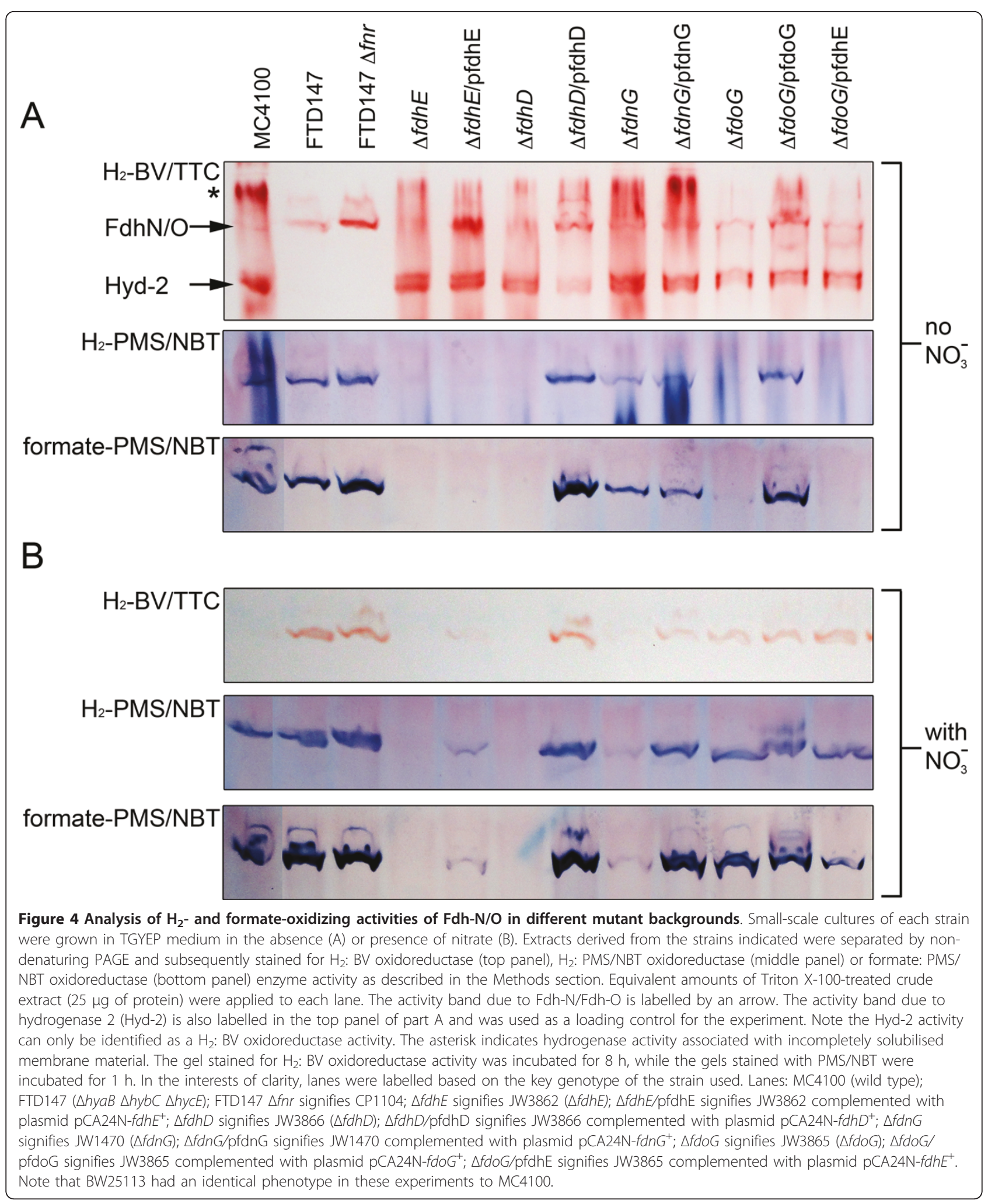


for formate: PMS/NBT oxidoreductase enzyme activity (Figure 4A, bottom panel) revealed that after growth under fermentative conditions, Fdh-O had a stronger contribution to the overall intensity of the activity band than Fdh-N, because while extracts derived from the $f d n G$ mutant had similar activity to the wild type, the activity in an extract from the fdoG mutant was considerably reduced. Introduction of the $f d o G$ gene on a plasmid, however, restored the activity to the mutant (Figure 4A bottom panel). Notably, replacing formate with hydrogen as electron donor revealed that both enzymes also catalyzed the hydrogen-dependent reduction of PMS/NBT (Figure 4A, middle panel). A similar pattern for $\mathrm{H}_{2}$ : PMS/NBT oxidoreductase activity was observed as was seen for formate: PMS/NBT oxidoreductase activity (compare the middle and bottom panels in Figure 4A). Taken together, these findings suggest that Fdh- $\mathrm{N}$ is the more effective enzyme at transferring the electrons from $\mathrm{H}_{2}$ to $\mathrm{BV} / \mathrm{TTC}$ than to PMS/NBT. That Fdh-O is nevertheless effective at catalyzing $\mathrm{H}_{2}$ dependent $\mathrm{BV}$ reduction is shown in the lane containing an extract derived from CP1104 (labelled FTD147 $\Delta f n r$ in Figure 4) in which an fnr mutation was introduced into the hydrogenase-negative strain FTD147 (Figure 4, top panel). Synthesis of Fdh-N is absolutely dependent on the redox regulator FNR $[1,21]$ and thus is absent in an $f u r$ mutant. In contrast, Fdh-O activity is apparently up-regulated in the fnr mutant (Figure 4A).

Fdh-N/O show $\mathrm{H}_{2}$ : $\mathrm{BV}$ and $\mathrm{H}_{2}$ : PMS/NBT oxidoreductase activities in extracts after respiratory growth with nitrate Biosynthesis of Fdh-N is enhanced when E. coli is grown anaerobically in the presence of nitrate $[1,5,21]$, while synthesis of Fdh-O is essentially constitutive [9]. The same strains analyzed in Figure 4A were grown anaerobically in the presence of nitrate and aliquots of crude extracts were separated by non-denaturing PAGE followed by staining for $\mathrm{H}_{2}$ : BV oxidoreductase, $\mathrm{H}_{2}$ : PMS/ NBT oxidoreductase and formate: PMS/NBT oxidoreductase activities. The gel presented in the top panel of Figure $4 \mathrm{~B}$ shows clearly a $\mathrm{H}_{2}$ : BV oxidoreductase activity in extracts of strains FTD147, CP1104 (FTD147 $f$ fnr), as well as in the $f d o G$ mutant. The activity in extracts of MC4100 shown in this experiment was only weakly discernable (Figure 4B, top panel, first lane). As anticipated [13], synthesis of Hyd-1 and Hyd-2 was strongly reduced in MC4100 after growth in the presence of nitrate (data not shown). The mutant with a deletion in the $f d n G$ gene essentially lacked $\mathrm{H}_{2}$ : BV oxidoreductase activity but this could be recovered by introduction of the $f d n G$ gene on plasmid pCA24N-fdnG ${ }^{+}$(Figure 4B, top panel).

Aliquots of the same extracts specifically stained to visualize $\mathrm{H}_{2}$ : PMS/NBT oxidoreductase and formate: PMS/NBT oxidoreductase activities showed a strong
Fdh-N-dependent $\mathrm{H}_{2}$ : PMS/NBT oxidoreductase activity (Figure 4B, middle panel). Substituting hydrogen with formate as electron donor delivered a similar staining pattern (Figure 4B, bottom panel).

\section{Hydrogen oxidation by Fdh- $\mathrm{N}$ and Fdh-O is dependent on the accessory proteins FdhD and FdhE}

The $f d o G H I$ operon encoding Fdh-O is flanked by $f d h D$ and $f d h E$, both of which encode accessory enzymes required for the synthesis of active Fdh enzymes [22,23]. To demonstrate the dependence of the $\mathrm{H}_{2}$-oxidizing activities of both Fdhs on FdhD and FdhE, individual mutants lacking either the $f d h D$ or the $f d h E$ gene were analyzed under the same conditions as described above for the wild type and $f d o G$ and $f d n G$ mutants. All three activities were absolutely dependent on both FdhD and FdhE (Figure 4). Complementation experiments revealed that while FdhD on a plasmid fully complemented the $f d h D$ mutation, plasmid-encoded FdhE only partially complemented the $f d h E$ mutation.

\section{Discussion}

We demonstrate here that both of the respiratory formate dehydrogenases Fdh- $\mathrm{N}$ and Fdh-O have hydrogenoxidizing enzyme activity. Together with the three characterized $[\mathrm{NiFe}]$-hydrogenases, these are the only two enzymes in E. coli crude extracts that had this activity. These results suggest that the Fdh-N and Fdh-O enzymes show a degree of non-specificity with regard to the electron donor they can use. Notably, formate and dihydrogen $\left(\mathrm{CO}_{2} /\right.$ formate, $\left.\mathrm{E}_{\mathrm{o}}{ }^{\prime}=-432 \mathrm{mV}[24]\right)$ and $(\mathrm{H}$ '/hydrogen, $E_{\mathrm{o}}{ }^{\prime}=-414 \mathrm{mV}$ ) are both strong reductants.

Previous studies have demonstrated that $E$. coli can couple hydrogen oxidation to nitrate reduction and Hyd-1 and Hyd-2 participate in this process [25]. However, attempts to demonstrate significant hydrogendependent nitrate reduction in the absence of Hyd-1 and Hyd-2 did not deliver reproducible levels of hydrogen oxidation, presumably due to the limited hydrogenoxidizing activity of Fdh-N and Fdh-O. Nevertheless, the findings reported here might have physiological relevance in other microorganisms. For example, enzymes with subunits orthologous to FdnG are found in the obligate dehalorespiring and hydrogen-oxidizing Dehalococcoides spp., e.g. strain CBDB1, and have an associated subunit with similarity to hydrogenase membraneanchoring subunits [26]. Rather than having a selenocysteinyl residue in their presumptive active site they have a seryl residue. It is established that in E. coli replacement of selenocysteine with serine abolishes the formate-oxidizing activity of Fdh-H [27]. Moreover, it is also clear that Dehalococcoides strain CBDB1 cannot use formate as a substrate, suggesting that this formate dehydrogenase-like enzyme might have another 
function. One possibility based on the findings presented here might be that it accepts $\mathrm{H}_{2}$ as substrate.

As both Fdh enzymes are selenium-dependent, impaired co-translational insertion of selenocysteine prevented synthesis of either enzyme and concomitantly abolished the [NiFe]-hydrogenase-independent $\mathrm{H}_{2}$ : BV oxidoreductase activity. Moreover, because both Fdh-N and Fdh-O are absolutely dependent on the FdhE and FdhD proteins for activity of the enzyme [22,23], deletion of $f d h E$ and $f d h D$ also abolished specifically this $\mathrm{H}_{2}$ : BV oxidoreductase activity. The precise functions of FdhD and FdhE in formate dehydrogenase biosynthesis remain to be established; however, it is likely that they perform a function in post-translational maturation of the enzymes [22].

While it is established that the iron-molybdenum cofactor in nitrogenase catalyzes unidirectional proton reduction as an inevitable consequence of nitrogen reduction [28], the studies here present the first report of a seleno-molybdenum enzyme catalyzing dihydrogen activation. Recent studies have shown that high-valence (oxidation state VI) oxo-molybdenum model complexes can activate dihydrogen at high temperature and $\mathrm{H}_{2}$ pressure [29]. The crystal structure of Fdh-N [4] also reveals a similar geometry of the molybdenum atom to these model complexes; however, along with the four cis thiolate groups, which are derived from the two MGD cofactors, a hydroxyl from a water molecule and the selenate group from selenocysteine coordinate the Mo atom. The coordination geometry might play an important role in conferring hydrogen activation capability, as the molybdoenzyme nitrate reductase from E. coli [30] cannot oxidize dihydrogen. Instead of the selenate ligand, nitrate reductase has an oxo ligand to the Mo, which is contributed by an aspartate residue. In this regard, however, it should be noted that although the third formate dehydrogenase Fdh-H also has similar active site geometry to Fdh- $\mathrm{N}[4,7]$, we could not detect a dihydrogen-activating activity associated with this enzyme in our gel system. In contrast to other molybdopterin-containing molybdoenzymes catalyzing oxotransfer of the oxygen from $\mathrm{H}_{2} \mathrm{O}$ to the substrate, Fdh$\mathrm{H}$, and presumably also Fdh-N and Fdh-O, catalyze the direct release of $\mathrm{CO}_{2}$ and not bicarbonate from formate [31]. The transfer of the proton from formate to a histidine and concomitant reduction of $\mathrm{Mo}(\mathrm{VI})$ to $\mathrm{Mo}(\mathrm{IV})$ facilitates direct release of $\mathrm{CO}_{2}$ with the cofactor returning to the oxidized $\mathrm{Mo}(\mathrm{VI})$ state after electron transfer to the iron-sulfur cluster [31]. Such a dehydrogenation reaction could explain the inefficient oxidation of $\mathrm{H}_{2}$ by Fdh-N/O demonstrated here. Future studies will focus on testing this hypothesis to characterize the mechanism of dihydrogen activation.

\section{Conclusions}

The energy-conserving formate dehydrogenases of $E$. coli can use dihydrogen as an enzyme substrate. Apart from the $[\mathrm{NiFe}]$-hydrogenases, these enzymes were the only ones in extracts of anaerobically grown $E$. coli that could oxidize hydrogen and transfer the electrons to benzyl viologen or phenazine methosulfate/nitroblue tetrazolium. While the possible significance of this activity to the general anaerobic physiology of $E$. coli remains to be established, this finding has potentially important implications for our understanding of the hydrogen metabolism of other anaerobic microorganisms.

\section{Methods}

\section{Strains, plasmids and growth conditions}

All bacterial strains and plasmids used in this study are listed in Table 2. Bacterial growth of all E. coli strains was performed at $37^{\circ} \mathrm{C}$. E. coli cells were cultivated anaerobically in buffered TYEP medium [32] supplemented with $0.8 \%(\mathrm{w} / \mathrm{v})$ glucose. Where indicated formate was added to a final concentration of $15 \mathrm{mM}$ and nitrate to $15 \mathrm{mM}$. Aerobic cultures were grown in flasks filled maximally to $10 \%$ of their volume, while anaerobic cultures were grown in stoppered bottles filled to the top with medium. When required, kanamycin was added to a final concentration of $50 \mu \mathrm{g} / \mathrm{ml}$ and chloramphenicol to a final concentration $15 \mu \mathrm{g} / \mathrm{ml}$. Cultures were harvested after reaching an optical density at 600 $\mathrm{nm}$ of 0.9 was attained. Cells were collected by

\section{Table 2 Strains and plasmids used in this study}

\begin{tabular}{|c|c|c|}
\hline Strains & Genotype & $\begin{array}{l}\text { Reference or } \\
\text { source }\end{array}$ \\
\hline MC4100 & $\begin{array}{l}\text { F }^{-} \text {araD139 } \Delta \text { (argF-lac)U169 ptsF25 } \\
\text { deoC1 } \\
\text { relA1 flbB5301 rspL150 }\end{array}$ & {$[38]$} \\
\hline MC-NG & Like MC4100, but $\Delta f d n G$ & This work \\
\hline MC-OG & Like MC4100, but $\triangle f d o G$ & This work \\
\hline FM460 & Like MC4100, but $\Delta s e / C$ & {$[34]$} \\
\hline DHP-F2 & Like MC4100, but $\Delta$ hypF & [17] \\
\hline FTD147 & Like MC4100, but $\triangle h y a B, \Delta h y b C, \Delta h y c E$ & [19] \\
\hline CP1104 & Like FTD147, but $\Delta$ fnr & This work \\
\hline JW1328 & BW25113 $\Delta f n r$ & {$[39]$} \\
\hline JW3862 & BW25113 $\Delta f d h E$ & [39] \\
\hline JW3866 & BW25113 $\Delta f d h D$ & [39] \\
\hline JW1470 & BW25113 $\Delta f d n G$ & [39] \\
\hline JW3865 & BW25113 $\Delta$ fdoG & [39] \\
\hline \multicolumn{3}{|c|}{ Plasmids } \\
\hline$\overline{p f d h E}$ & pCA24N fdhE & {$[39]$} \\
\hline pfdhD & pCA24N fdhD ${ }^{+}$ & {$[39]$} \\
\hline pfdnG & $\mathrm{pCA} 24 \mathrm{~N} f d n G^{+}$ & [39] \\
\hline pfdoG & $\mathrm{pCA} 24 \mathrm{~N}$ fdo $\mathrm{G}^{+}$ & [39] \\
\hline
\end{tabular}


centrifugation at 50,000 xg for $20 \mathrm{~min}$ at $4^{\circ} \mathrm{C}$. Harvested cell pellets were suspended in $50 \mathrm{ml} 50 \mathrm{mM}$ MOPS pH 7.5 and re-centrifuged under the same conditions. Washed cell pellets were either used immediately or stored at $-20^{\circ} \mathrm{C}$ until use.

\section{Strain construction}

Deletions in the $f d n G$ and $f d o G$ genes were introduced into appropriate strains by $\mathrm{P} 1_{k c}$ transduction [33] using strains JW1470 $\left(\Delta f d n G:: \operatorname{Kan}^{\mathrm{R}}\right)$ or JW3865 $\left(\Delta f d o G:: \operatorname{Kan}^{\mathrm{R}}\right)$ (obtained from the National BioResources Project, Japan) as donors. The selC mutation from FM460 [34] was moved in a similar manner into clean genetic backgrounds. Similarly, the $f n r$ mutation from JW1328 was transduced into FTD147 to create FTD147 $\Delta$ fnr.

\section{Measurement of enzyme activity}

Hydrogen-dependent reduction of benzyl viologen (referred to as hydrogenase activity) was determined as described [12] using $50 \mathrm{mM}$ sodium phosphate $\mathrm{pH}$ 7.2. One unit of enzyme activity is defined as that which reduces $1 \mu \mathrm{mol}$ of dihydrogen $\mathrm{min}^{-1}$. Formate dehydrogenase enzyme activity was assayed spectrophotometrically at RT by monitoring the formate-dependent, PMSmediated reduction of 2, 6- dichlorophenolindophenol (DCPIP) exactly as described [35] or the formate-dependent reduction of benzyl viologen. The latter assay was performed exactly as for the hydrogenase assay with the exception that $50 \mathrm{mM}$ formate replaced hydrogen as enzyme substrate. One unit of enzyme activity is defined as that which oxidizes $1 \mu \mathrm{mol}$ of formate $\mathrm{min}^{-1}$.

Protein concentration was determined [36] with bovine serum albumin as standard.

\section{In-gel visualization of enzyme activity}

Detection of hydrogenase enzyme activity after nondenaturing PAGE was performed as described [12]. Samples of crude extract or fractions after Q-sepharose, phenyl sepharose and Superdex 200 (5 to $50 \mu \mathrm{g}$ of protein) were incubated with $4 \%(\mathrm{v} / \mathrm{v})$ Triton X-100 for 30 min prior to application to the gels. After electrophoretic separation of the proteins, the gels were incubated in $50 \mathrm{mM}$ MOPS pH 7.2 containing $0.5 \mathrm{mM} \mathrm{BV}$ and 1 $\mathrm{mM} \mathrm{2,3,5-triphenyltetrazolium} \mathrm{chloride} \mathrm{and} \mathrm{they} \mathrm{were}$ incubated under a hydrogen: nitrogen atmosphere $(5 \%$ $\mathrm{H}_{2}: 95 \% \mathrm{~N}_{2}$ ) at room temperature for $8 \mathrm{~h}$. This assay was used to identify the hydrogen-oxidizing activity during the enrichment procedure described below.

Visualization of formate dehydrogenase enzyme activity was performed exactly as described [8] using phenazine methosulfate as mediator and nitroblue tetrazolium as electron acceptor. Visualization of the hydrogen: PMS/NBT oxidoreductase activity associated with Fdh$\mathrm{N}$ and Fdh-O was performed exactly for formate dehydrogenase but formate was replaced by hydrogen gas as enzyme substrate.

\section{Preparation of cell extracts and enrichment of the hydrogenase-independent hydrogen-oxidizing activity}

Unless indicated otherwise, all steps were carried out under anaerobic conditions in a Coy ${ }^{\mathrm{TM}}$ anaerobic chamber under a $\mathrm{N}_{2}$ atmosphere $\left(95 \% \mathrm{~N}_{2}: 5 \% \mathrm{H}_{2}\right)$ and at $4{ }^{\circ} \mathrm{C}$. All buffers were boiled, flushed with $\mathrm{N}_{2}$, and maintained under a slight overpressure of $\mathrm{N}_{2}$.

For routine experiments and enzyme assay determination, washed cells ( $1 \mathrm{~g}$ wet weight) were resuspended in $3 \mathrm{ml}$ of $50 \mathrm{mM}$ MOPS pH 7.5 including $5 \mu \mathrm{g}$ DNase $/ \mathrm{ml}$ and $0.2 \mathrm{mM}$ phenylmethylsulfonyl fluoride. Cells were disrupted by sonication (30W power for 5 min with 0.5 sec pulses). Unbroken cell and cell debris were removed by centrifugation for $30 \mathrm{~min}$ at $50000 \mathrm{xg}$ at $4^{\circ} \mathrm{C}$ and the supernatant (crude extract) was decanted. Small-scale analyses were carried out with 0.1-0.2 g wet weight of cells suspended in a volume of $1 \mathrm{ml}$ MOPS buffer as described above. Cell disruption was done by sonication as described above.

To enrich the protein(s) responsible for the hydrogenase-independent hydrogen-oxidizing activity, crude membranes were isolated from cell extracts routinely prepared from $20 \mathrm{~g}$ (wet weight) of cells by ultracentrifugation at $145000 \times \mathrm{g}$ for $2 \mathrm{~h}$. Crude membranes were then suspended in $60 \mathrm{ml}$ of $50 \mathrm{mM}$ MOPS, pH 7.5 (buffer A). Triton X-100 was added to the suspended membrane fraction to a final concentration of $4 \%(\mathrm{v} / \mathrm{v})$ and the mixture was incubated for $4 \mathrm{~h}$ at $4^{\circ} \mathrm{C}$ with gentle swirling. After centrifugation at $145000 \mathrm{xg}$ for $1 \mathrm{~h}$ to remove insoluble membrane particles, the solubilized membrane proteins present in the supernatant were loaded onto a Q-Sepharose HiLoad column (2.6 x15 $\mathrm{cm})$ equilibrated with buffer A. Unbound protein was washed from the column with $60 \mathrm{ml}$ of buffer A. Protein was eluted from the column with a stepwise $\mathrm{NaCl}$ gradient $(80 \mathrm{ml}$ each of $0.1 \mathrm{M}, 0.2 \mathrm{M}, 0.3 \mathrm{M}, 0.4 \mathrm{M}, 0.5 \mathrm{M}$ and $1 \mathrm{M}$ ) in buffer $\mathrm{A}$ at a flow rate of $5 \mathrm{ml} \mathrm{min}^{-1}$. Activity was recovered in the fractions eluting with $0.4 \mathrm{M}$ $\mathrm{NaCl}$. The fractions containing enzyme activity were brought to a concentration of $0.5 \mathrm{M}$ ammonium sulfate and loaded onto a hydrophobic interaction chromatography column (Phenyl-Sepharose HiLoad; $2.6 \times 10 \mathrm{~cm}$ ) equilibrated with $0.5 \mathrm{M}$ ammonium sulfate in buffer $\mathrm{A}$. Protein was eluted using a stepped ammonium sulfate gradient $(60 \mathrm{ml}$ each of $0.4 \mathrm{M}, 0.3 \mathrm{M}, 0.2 \mathrm{M}, 0.1 \mathrm{M}$ and without ammonium sulfate) in buffer $\mathrm{A}$ and at a flow rate of $5 \mathrm{ml} \mathrm{min}^{-1}$. The hydrogen-oxidizing activity was recovered in the fractions eluting with only buffer $\mathrm{A}$. Fractions containing enzyme activity were concentrated by centrifugation at 7,500 $\times \mathrm{g}$ in centrifugal filters (Amicon Ultra, $50 \mathrm{~K}$, Millipore, Eschborn, Germany) and 
applied to a Hi-Load Superdex-200 gel filtration column $(2.6 \times 60 \mathrm{~cm})$ equilibrated with buffer A containing 0.1 $\mathrm{M} \mathrm{NaCl}$. Fractions containing the hydrogen-oxidizing activity eluted after $47 \mathrm{ml}$ (peak maximum); the void volume $\mathrm{V}_{\mathrm{o}}$ of the column was $45 \mathrm{ml}$ and the separation range was from 60-600 kDa. Protein was stored in buffer A containing $0.1 \mathrm{M} \mathrm{NaCl}$ at a concentration of $3 \mathrm{mg}$ protein $\mathrm{ml}^{-1}$. The activity was stable for several months when stored at $-80^{\circ} \mathrm{C}$.

\section{Mass spectrometric identification of proteins}

For mass spectrometric analysis the gel band showing $\mathrm{H}_{2}$ : BV oxidoreductase activity after hydrophobic interaction chromatography was excised and the proteins within the band were in-gel digested following standard protocols [37]. Briefly, protein disulfides were reduced with DTT and cysteines were alkylated with iodoacetamide. Digestion was performed at $37^{\circ} \mathrm{C}$ for two hours using trypsin as protease. ProteaseMax ${ }^{\circledR}$ surfactant was used in the digestion and extraction solutions to improve the recovery of hydrophobic peptides. The peptide extracts were analyzed by LC/MS on an UltiMate Nano-HPLC system (LC Packings/Dionex) coupled to an LTQ-Orbitrap XL mass spectrometer (ThermoFisher Scientific) equipped with a nanoelectrospray ionization source (Proxeon). The samples were loaded onto a trapping column (Acclaim PepMap C18, $300 \mu \mathrm{m} \times 5 \mathrm{~mm}, 5 \mu \mathrm{m}, 100 \AA$, LC Packings) and washed for 15 min with $0.1 \%$ trifluoroacetic acid at a flow rate of $30 \mu \mathrm{l} / \mathrm{min}$. Trapped peptides were eluted using a separation column (Acclaim PepMap C18, 75 $\mu \mathrm{m} \times 150 \mathrm{~mm}, 3 \mu \mathrm{m}, 100 \AA$, LC Packings) that had been equilibrated with $100 \%$ A (5\% acetonitrile, $0.1 \%$ formic acid). Peptides were separated with a linear gradient: $0-50 \%$ B (80\% acetonitrile, $0.1 \%$ formic acid) in $90 \mathrm{~min}, 50-100 \%$ B in $1 \mathrm{~min}$, remain at $100 \%$ B for 5 min. The column was kept at $30^{\circ} \mathrm{C}$ and the flow-rate was $300 \mathrm{nl} / \mathrm{min}$. During the duration of the gradient, online MS data were acquired in data-dependent MS/ MS mode: Each high-resolution full scan $(\mathrm{m} / \mathrm{z} 300$ to 2000 , resolution 60,000 ) in the orbitrap analyzer was followed by five product ion scans (collision-induced dissociation (CID)-MS/MS) in the linear ion trap for the five most intense signals of the full scan mass spectrum (isolation window $2 \mathrm{Th}$ ). Both precursor and fragment ions were analyzed in the orbitrap analyzer. Dynamic exclusion (repeat count was 3, exclusion duration $180 \mathrm{~s}$ ) was enabled to allow detection of less abundant ions. Data analysis was performed using the Proteome Discoverer 1.0 (Thermo Fisher Scientific), MS/MS data of precursor ions in the $m / z$ range 3508000 were searched against the SwissProt Database (version 53.3, taxonomy E. coli, 8,852 entries) using Mascot (version 2.2, Matrixscience), mass accuracy was set to $3 \mathrm{ppm}$ and $0.01 \mathrm{Da}$ for precursor and fragment ions, respectively. Carbamidomethylation of cysteines was set as static modification and oxidation of methionine as potential modification. Up to four missed cleavages of trypsin were allowed. Proteins identified by at least two peptides with an expectation value $<0.01$ were considered as unambiguously identified.

\section{Acknowledgements}

This work was supported by the Deutsche Forschungsgemeinschaft (SA 494/ 3-1 and SA 494/6-1 to RGS; SI 867/13-1 and SI 867/15-1 to AS), the Region of Saxony-Anhalt (to RGS \& AS), and the BMBF (ProNet-T3, Project To-06 to AS). KT was the recipient of a short-term FEBS Summer Research Fellowship.

\section{Author details}

${ }^{1}$ Institute for Microbiology, Martin-Luther University Halle-Wittenberg, KurtMothes-Str. 3, 06120 Halle (Saale), Germany. ${ }^{2}$ Institute of Pharmacy, MartinLuther University Halle-Wittenberg, Wolfgang-Langenbeck-Str. 106120 Halle (Saale), Germany. ${ }^{3}$ Department of Biophysics, Yerevan State University, 1 A. Manoukian Str., Yerevan 0025, Armenia.

\section{Authors' contributions}

BS, MK, MW, CP and KT carried out the biochemical studies. $\mathrm{Cl}$ performed the mass spectrometric analyses and $\mathrm{Cl}$ and $\mathrm{AS}$ interpreted the data. BS, CP, AT, AS and RGS conceived the study and helped draft the manuscript. RGS wrote the manuscript. All authors have read and approved the manuscript.

\section{Competing interests}

The authors declare that they have no competing interests.

Received: 10 June 2011 Accepted: 1 August 2011

Published: 1 August 2011

\section{References}

1. Sawers G: The hydrogenases and formate dehydrogenases of Escherichia coli. Antonie van Leeuvenhoek 1994, 66:57-88.

2. Sawers $G$, Blokesch M, Böck A: Anaerobic formate and hydrogen metabolism. In EcoSal- Escherichia coli and Salmonella: Cellular and Molecular Biology. Edited by: Curtiss III R.(Editor in Chief). ASM Press, Washington, D.C; [http://www.ecosal.org], September 2004, posting date.

3. Sawers RG: Formate and its role in hydrogen production in Escherichia coli. Biochem Soc Trans 2005, 33:42-46.

4. Jormakka M, Törnroth S, Byrne B, Iwata S: Molecular basis of proton motive force generation: structure of formate dehydrogenase-N. Science 2002, 295:1863-1868

5. Berg BL, Li J, Heider J, Stewart V: Nitrate-inducible formate dehydrogenase in Escherichia coli K-12. I. Nucleotide sequence of the $f d n G H I$ operon and evidence that opal (UGA) encodes selenocysteine. $J$ Biol Chem 1991, 266:22380-22385.

6. Abaibou H, Pommier J, Benoit S, Giordano G, Mandrand-Berthelot MA: Expression and characterization of the Escherichia coli fdo locus and a possible physiological role for aerobic formate dehydrogenase. $J$ Bacteriol 1995, 177:7141-7149.

7. Boyington JC, Gladyshev VN, Khangulov SV, Stadtman TC, Sun PD: Crystal structure of formate dehydrogenase $\mathrm{H}$ : catalysis involving Mo, molybdopterin, selenocysteine, and an $\mathrm{Fe}_{4} \mathrm{~S}_{4}$ cluster. Science 1997, 275:1305-1308.

8. Enoch $H G$, Lester RL: The purification and properties of formate dehydrogenase and nitrate reductase from Escherichia coli. J Biol Chem 1975, 250:6693-6705.

9. Sawers G, Heider J, Zehelein E, Böck A: Expression and operon structure of the sel genes of Escherichia coli and identification of a third seleniumcontaining formate dehydrogenase isoenzyme. J Bacteriol 1991, 173:4983-4993.

10. Forzi L, Sawers RG: Maturation of [NiFe]-hydrogenases in Escherichia coli. Biometals 2007, 20:565-578

11. Sargent F: Constructing the wonders of the bacterial world: biosynthesis of complex enzymes. Microbiology 2007, 153:633-651. 
12. Ballantine SP, Boxer DH: Nickel-containing hydrogenase isoenzymes from anaerobically grown Escherichia coli K-12. J Bacteriol 1985, 163:454-459.

13. Sawers RG, Ballantine SP, Boxer DH: Differential expression of hydrogenase isoenzymes in Escherichia coli K-12: evidence for a third isoenzyme. J Bacteriol 1985, 164:1324-1331.

14. Sawers RG: Membrane-bound hydrogenase isoenzymes from Escherichia coli. PhD Thesis University of Dundee; 1985.

15. Sawers RG, Boxer DH: Purification and properties of membrane-bound hydrogenase isoenzyme 1 from anaerobically grown Escherichia coli K12. Eur J Biochem 1986, 156:265-275.

16. Pinske C, Sawers RG: The role of the ferric-uptake regulator Fur and iron homeostasis in controlling levels of the [NiFe]-hydrogenases in Escherichia coli. Int J Hydrogen Energy 2010, 35:8938-8944.

17. Paschos A, Bauer A, Zimmermann A, Zehelein E, Böck A: HypF, a carbamoyl phosphate-converting enzyme involved in [NiFe] hydrogenase maturation. J Biol Chem 2002, 277:49945-4995.

18. Böck A, Forchhammer K, Heider J, Baron C: Selenoprotein synthesis: an expansion of the genetic code. Trends Biochem Sci 1991, 16:463-467.

19. Leinfelder W, Zehelein E, Mandrand-Berthelot M-A, Böck A: Gene for a novel tRNA species that accepts L-serine and co-translationally inserts selenocysteine. Nature 1988, 331:723-725

20. Redwood MD, Mikheenko IP, Sargent F, Macaskie LE: Dissecting the roles of Escherichia coli hydrogenases in biohydrogen production. FEMS Microbiol Lett 2008, 278:48-55.

21. Berg BL, Stewart V: Structural genes for nitrate-inducible formate dehydrogenase in Escherichia coli K-12. Genetics 1990, 125:691-702.

22. Lüke I, Butland G, Moore K, Buchanan G, Lyall V, Fairhurst SA, Greenblatt JF, Emili A, Palmer T, Sargent F: Biosynthesis of the respiratory formate dehydrogenases from Escherichia coli: characterization of the FdhE protein. Arch Microbiol 2010, 190:685-696.

23. Schlindwein C, Giordano G, Santini CL, Mandrand MA: Identification and expression of the Escherichia coli fdhD and fdhE genes, which are involved in the formation of respiratory formate dehydrogenase. $J$ Bacteriol 1993, 172:6112-6121.

24. Thauer RK, Jungermann K, Decker K: Energy conservation in chemotrophic anaerobic bacteria. Bacteriol Rev 1977, 41:100-180.

25. Laurinavichene TV, Tsygankov AA: The involvement of hydrogenases and 2 in the hydrogen-dependent nitrate respiration of Escherichia coli. Microbiology (Mikrobiologiya, Russia) 2003, 72:740-745.

26. Kube $\mathrm{M}$, Zinder $\mathrm{SH}$, Kuhl H, Reinhardt R, Adrian L: Genome sequence of the chlorinated compound-respiring bacterium Dehalococcoides species strain CBDB1. Nature Biotechnol 2005, 23:1269-1273.

27. Zinoni $F$, Birkmann $A$, Leinfelder W, Böck A: Cotranslational insertion of selenocysteine into formate dehydrogenase from Escherichia coli directed by a UGA codon. Proc Natl Acad Sci USA 1987, 84:3156-3160.

28. Seefeldt LC, Hoffman BM, Dean DR: Mechanism of Mo-dependent nitrogenase. Annu Rev Biochem 2009, 78:701-722.

29. Reis PM, Paulo Costa J, Romão CC, Fernandes JA, Calhorda MJ, Royo B: Hydrogen activation by high-valent oxo-molybdenum(VI) and -rhenium (VII) and -(V) compounds. Dalton Trans 2008, 13:1727-1733.

30. Bertero MG, Rothery RA, Palak M, Hou C, Lim D, Blasco F, Weiner JH, Strynadka NCJ: Insights into the respiratory electron transfer pathway from the structure of nitrate reductase A. Nature Struct Biol 2003, 10:681-687.

31. Khangulov SV, Gladyshev VN, Dismukes GC, Stadtman TC: Seleniumcontaining formate dehydrogenase $\mathrm{H}$ from Escherichia coli: a molybdopterin enzyme that catalyzes formate oxidation without oxygen transfer. Biochemistry 1998, 37:3518-3528.

32. Begg YA, Whyte JN, Haddock BA: The identification of mutants of Escherichia coli deficient in formate dehydrogenase and nitrate reductase activities using dye indicator plates. FEMS Microbiol Lett 1977 2:47-50.

33. Miller JH: Experiments in Molecular Genetics Cold Spring Harbor, Cold Spring Harbor Press; 1972

34. Leinfelder W, Forchhammer K, Zinoni F, Sawers G, Mandrand-Berthelot M-A, Böck A: Escherichia coli genes whose products are involved in selenium metabolism. J Bacteriol 1988, 170:540-546.

35. Lester RL, DeMoss JA: Effects of molybdate and selenite on formate and nitrate metabolism in Escherichia coli. J Bacteriol 1971, 105:1006-1014.

36. Lowry OH, Rosebrough NJ, Farr AL, Randall RJ: Protein measurement with the Folin phenol reagent. J Biol Chem 1951, 193:265-275.
37. Shevchenko A, Tomas H, Havliš J, Olsen JV, Mann M: In-gel digestion for mass spectrometric characterization of proteins and proteomes. Nature Protocols 2007, 1:2856-60.

38. Casadaban MJ: Transposition and fusion of the lac genes to selected promoters in Escherichia coli using bacteriophage lambda and $\mathrm{Mu}$. J Mol Biol 1976, 104:541-555

39. Kitagawa M, Ara T, Arifuzzaman M, loka-Nakamichi T, Inamoto $\mathrm{E}$, et al: Complete set of ORF clones of Escherichia coli ASKA library (a complete set of $E$. coli $\mathrm{K}-12$ ORF archive): unique resources for biological research. DNA Res 2005, 12:291-299.

\section{doi:10.1186/1471-2180-11-173}

Cite this article as: Soboh et al:: The respiratory molybdo-selenoprotein formate dehydrogenases of Escherichia coli have hydrogen: benzyl viologen oxidoreductase activity. BMC Microbiology 2011 11:173.

\section{Submit your next manuscript to BioMed Central and take full advantage of:}

- Convenient online submission

- Thorough peer review

- No space constraints or color figure charges

- Immediate publication on acceptance

- Inclusion in PubMed, CAS, Scopus and Google Scholar

- Research which is freely available for redistribution
Ciomed Central 